\title{
Prominence seismology using the period ratio of transverse thread oscillations
}

\author{
R. Soler ${ }^{1}$, M. Goossens ${ }^{2}$, and J. L. Ballester ${ }^{1}$ \\ 1 Departament de Física, Universitat de les Illes Balears, 07122 Palma de Mallorca, Spain \\ e-mail: roberto.soler@uib.es \\ 2 Centre for mathematical Plasma Astrophysics, Department of Mathematics, KU Leuven, Celestijnenlaan 200B, 3001 Leuven, \\ Belgium \\ e-mail: marcel .goossens@wis. kuleuven . be
}

Received 14 May 2014 / Accepted 21 January 2015

\begin{abstract}
The ratio of the period of the fundamental mode to that of the first overtone of kink oscillations (hereafter period ratio) is a seismology tool that can be used to infer information about the spatial variation of density along solar magnetic flux tubes. The period ratio is 2 in longitudinally homogeneous thin tubes, but it differs from 2 because of longitudinal inhomogeneity. In this paper we investigate the period ratio in longitudinally inhomogeneous prominence threads and explore its implications for prominence seismology. We numerically solve the two-dimensional eigenvalue problem of kink oscillations in a model of a prominence thread. We take into account three nonuniform density profiles along the thread. In agreement with previous works that used simple piecewise constant density profiles, we find that the period ratio is larger than 2 in prominence threads. When the ratio of the central density to that at the footpoints is fixed, the period ratio depends strongly on the form of the density profile along the thread. The more concentrated the dense prominence plasma near the center of the tube, the larger the period ratio. However, the period ratio is found to be independent of the specific density profile when the spatially averaged density in the thread is the same for all the profiles. An empirical fit of the dependence of the period ratio on the average density is given and its use for prominence seismology is discussed.
\end{abstract}

Key words. magnetohydrodynamics (MHD) - Sun: atmosphere - Sun: filaments, prominences - Sun: oscillations - waves

\section{Introduction}

High-resolution observations reveal that solar prominences are formed by a myriad of long and thin substructures usually called threads or fibrils. In observations of prominences above the limb, vertical threads are commonly seen in quiescent prominences (e.g., Berger et al. 2008), while horizontal threads are usually observed in active region prominences (e.g., Okamoto et al. 2007). However, it has been suggested (e.g., Schmieder et al. 2010) that vertical threads might actually be a pileup of horizontal threads that appear as vertical structures when projected on the plane of the sky. Along this line of thought, threads supported in quasi-horizontal magnetic fields seem to be more consistent with observations of filament threads on the solar disk (see Lin 2011), with determinations of the magnetic field orientation in prominences (e.g., Casini et al. 2003; Orozco Suárez et al. 2014a), and with equilibrium models of prominences based on slightly dipped magnetic fields (e.g., Terradas et al. 2013). In this work we use the term "thread" to refer to prominence horizontal fine structures.

Transverse oscillations of prominence threads with periods roughly between 1 and $20 \mathrm{~min}$ have frequently been reported (see, e.g., Okamoto et al. 2007; Lin et al. 2007, 2009; Ning et al. 2009; Orozco Suárez et al. 2014b). From the theoretical point of view, the oscillations are usually interpreted as magnetohydrodynamic (MHD) kink modes of the flux tube that supports the prominence thread (see, e.g., Terradas et al. 2008; Lin et al. 2009; Soler et al. 2010, 2012; Arregui et al. 2011; Soler \& Goossens 2011). Both observational and theoretical aspects of prominence thread oscillations have been reviewed by Arregui et al. (2012b).

Prominence seismology relies on the comparison of observed with predicted properties of prominence oscillations (see Ballester 2014). The predicted properties are based on theoretical models. The aim is to indirectly infer information about the plasma and/or the magnetic field in prominences (see, e.g., Terradas et al. 2008; Lin et al. 2009; Soler et al. 2010; Arregui et al. 2012a). In this direction, the ratio of the period of the fundamental longitudinal mode to the period of the first longitudinal overtone of thread kink oscillations (hereafter called the period ratio) can be used to obtain information about the spatial variation of density along the threads. Standing MHD waves on magnetic flux tubes can be characterized by the number of nodes in their eigenfunctions, and this classification is independent of the velocity polarization of the wave. Modes can have a different number of nodes in the longitudinal (axial) part of the eigenfunctions as well as in the radial part of the eigenfunctions. The terms fundamental longitudinal mode and first longitudinal overtone refer to waves that have no nodes and have only one node, respectively, in the longitudinal (axial) part of the eigenfunction. In the present work we study the fundamental longitudinal mode and the first longitudinal overtone of transverse kink waves. The period ratio is 2 in longitudinally homogeneous thin tubes, but it differs from 2 because of longitudinal inhomogeneity (Andries et al. 2005b,a). In the context of coronal loop transverse oscillations, the use of the period ratio as a seismology tool was first proposed by Andries et al. (2005a) and has been exploited in a number of subsequent works 


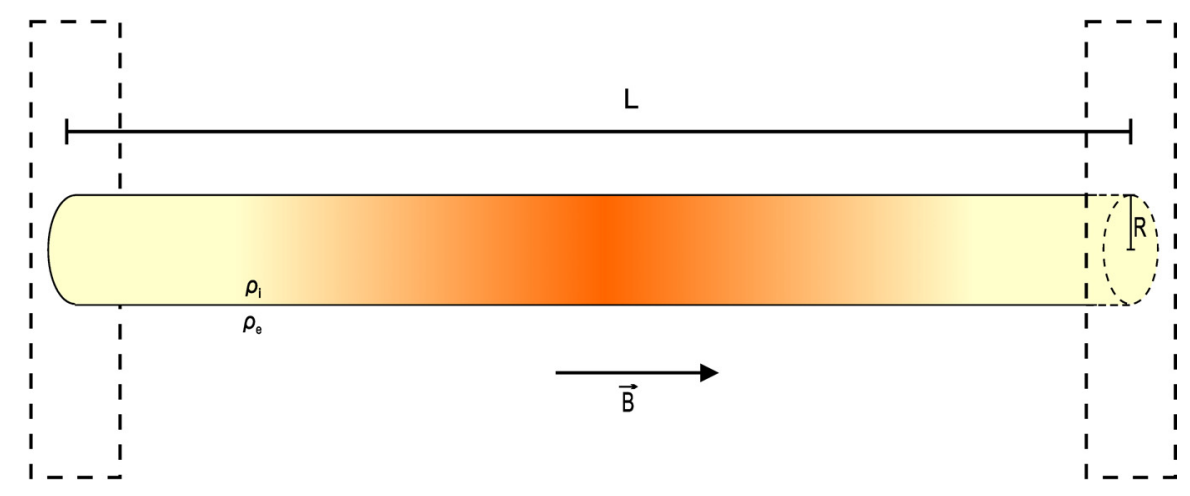

Fig. 1. Prominence thread model used in this work.

(e.g., McEwan et al. 2006, 2008; Dymova \& Ruderman 2006; Donnelly et al. 2007; Van Doorsselaere et al. 2007; Verth \& Erdélyi 2008; Ruderman et al. 2008; Arregui et al. 2013, among others). An extensive review of the use of the period ratio for coronal loop seismology can be found in Andries et al. (2009).

Although no reliable simultaneous observations of the two periods in prominence threads are currently available, numerical simulations of thread oscillations by Soler \& Goossens (2011) indicate that the fundamental mode and the first overtone are the modes that are more easily excited in threads as a result of external disturbances. It is reasonable to expect that the two periods will be eventually detected in prominence threads by future high-resolution observations. Because of its great potentiality for seismology, a firm theoretical basis on the value of the period ratio in prominence threads is needed. The use of the period ratio for prominence seismology was first studied by Díaz et al. (2010). They considered a prominence thread model composed of a thin magnetic flux tube filled with a dense blob of plasma that only occupied a small part of the tube, while the rest of the tube was evacuated, i.e., it was occupied by plasma that was much less dense. The density in both the dense blob and in the evacuated part was homogeneous, and an abrupt jump between the two densities was assumed. Similar piecewise constant models have been used in previous works as, e.g., Díaz et al. (2002), Dymova \& Ruderman (2005), Terradas et al. (2008), Soler et al. (2010, 2012), Soler \& Goossens (2011), while a continuous but thin transition between the dense blob and the evacuated part of the tube was considered by Arregui et al. (2011). The simple piecewise constant model used by Díaz et al. (2010) allowed these authors to derive analytic expressions for the periods of the fundamental mode and the first overtone. They found that, contrary to the case of coronal loops where the period ratio is smaller than 2 because of stratification, in prominence threads the period ratio is larger than 2 . The reason for this opposite result is that in prominence threads the densest plasma is located near the tube center, while in stratified coronal loops the plasma is denser near the footpoints. Subsequently, Soler \& Goossens (2011) added longitudinal flow to the model of Díaz et al. (2010) and found corrections to their results (see also Erdélyi et al. 2014).

The purpose of this paper is to advance the study of the period ratio of kink oscillations in inhomogeneous prominence threads and to explore its implications for prominence seismology. Here we go beyond the piecewise constant density profile used by Díaz et al. (2010) and consider a more realistic continuous variation of density along the thread. Assuming that the thread formation is due to condensation of cool plasma via thermal instability (see, e.g., Luna et al. 2012), the density profile along the threads is likely to depend on the energy balance between footpoint heating, radiative losses, and thermal conduction. This balance naturally leads to a spatially inhomogeneous density along the threads. Here, we evaluate the impact of the form of the longitudinal density profile on the period ratio. To do so, we numerically solve the full two-dimensional (2D) eigenvalue problem of kink oscillations in a longitudinally inhomogeneous prominence thread model.

This paper is organized as follows. Section 2 contains the description of the prominence thread model and the numerical method used to solve the eigenvalue problem of kink oscillations. The results of the computations are given in Sect. 3. The implications of the findings of this paper for prominence seismology are discussed in Sect. 4. Finally, concluding remarks are given in Sect. 5 .

\section{Prominence thread model and numerical solution of the eigenvalue problem}

The equilibrium model of a prominence thread is schematically shown in Fig. 1. We use a cylindrical coordinate system, with $r$, $\varphi$, and $z$ the radial, azimuthal, and longitudinal coordinates, respectively. We consider a cylindrically symmetric straight magnetic flux tube of radius $R$ and length $L$. The ends of the tube are located at $z= \pm L / 2$ and are line-tied at two rigid walls representing the solar photosphere. The center of the tube corresponds to $z=0$. The magnetic field, $\boldsymbol{B}$, is straight and along the axis of the tube. The magnetic field strength, $B_{0}$, is constant everywhere. We use the $\beta=0$ approximation, where $\beta$ refers to the ratio of the gas pressure to the magnetic pressure. This is an appropriate approximation to study kink oscillations, which are mainly driven by magnetic tension in thin tubes (Goossens et al. 2009). In the $\beta=0$ approximation we can freely choose the spatial distribution of mass density. Hence, the equilibrium density, $\rho_{0}$, is

$\rho_{0}(r, z)= \begin{cases}\rho_{\mathrm{i}}(z), & \text { if } r \leq R, \\ \rho_{\mathrm{e}}(z), & \text { if } r>R,\end{cases}$

where the internal, $\rho_{\mathrm{i}}(z)$, and external, $\rho_{\mathrm{e}}(z)$, densities are functions of $z$ alone, and $\rho_{\mathrm{i}}(z)>\rho_{\mathrm{e}}(z)$. The external plasma represents the coronal medium. In the present model the density jumps abruptly at the boundary of the tube, i.e., at $r=R$. We are aware that a continuous transition of density in the radial direction would be more realistic, although it would complicate matters. The kink oscillations of a transversely nonuniform tube are resonantly coupled to Alfvén waves and, consequently, the oscillations are damped in time (see, e.g., Goossens et al. 2011). In such a case, the kink mode is no longer a true normal mode of the flux tube, but a spectral pole of the Green's function, related to the initial-value problem, which represents a damped 


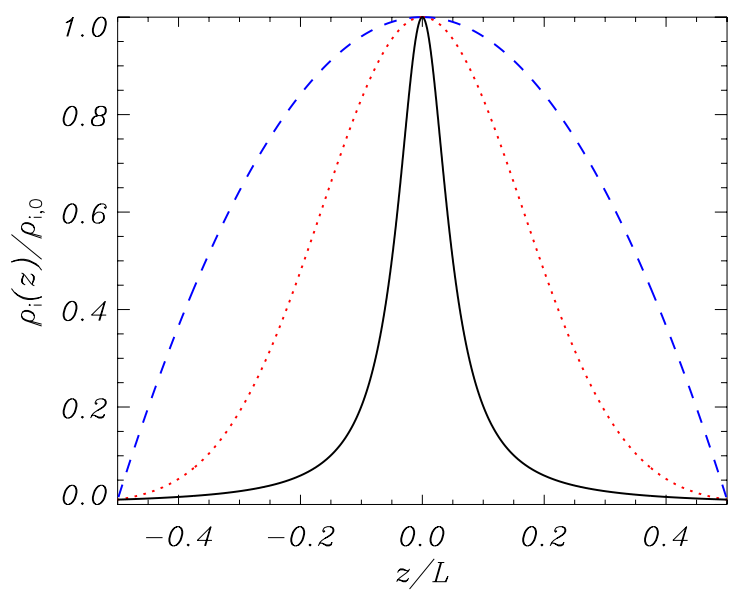

Fig. 2. Variation of the internal density along the prominence thread for the Lorentzian profile (black solid line), the Gaussian profile (red dotted line), and the parabolic profile (blue dashed line). We used $\chi=100$ for representation purposes.

coordinated motion of the plasma (Sedláček 1971). Since in the present work we are not interested in the damping, we assume a jump of density at the boundary of the tube to avoid the resonant absorption process. Readers are referred to Arregui et al. (2008, $2011)$ and Soler et al. $(2009,2010)$ for more information about the resonant damping of prominence thread kink oscillations and to Goossens et al. (2011) for a general review. A comparison of the efficiency of several damping mechanisms of transverse thread oscillations is given in Soler et al. (2014).

We denote the internal density at the center and at the end of the flux tube as $\rho_{\mathrm{i}}(0)=\rho_{\mathrm{i}, 0}$ and $\rho_{\mathrm{i}}(L / 2)=\rho_{\mathrm{i}, L / 2}$, respectively. Then, we define the ratio of the two densities as $\chi=\rho_{\mathrm{i}, 0} / \rho_{\mathrm{i}, L / 2}$, with $\chi \geq 1$. Contrary to coronal loops, prominence threads are denser near the center of the thread than at their footpoints. The larger $\chi$, the stronger the density variation along the thread, with the case $\chi=1$ corresponding to a homogeneous thread. Here, we consider three different longitudinal profiles for the internal density, namely a Lorentzian profile

$\rho_{\mathrm{i}}(z)=\frac{\rho_{\mathrm{i}, 0}}{1+4(\chi-1) z^{2} / L^{2}}$,

a Gaussian profile

$\rho_{\mathrm{i}}(z)=\rho_{\mathrm{i}, 0} \exp \left[-4(\log \chi) \frac{z^{2}}{L^{2}}\right]$,

and a parabolic profile

$\rho_{\mathrm{i}}(z)=\rho_{\mathrm{i}, 0}\left(1-4 \frac{\chi-1}{\chi} \frac{z^{2}}{L^{2}}\right)$.

The three density profiles are compared in Fig. 2. The three profiles represent different plasma arrangements within the magnetic tube. In the Lorentzian profile the densest plasma is narrowly concentrated near the center of the tube, while the rest of the tube is occupied by plasma that is much less dense. In essence, the Lorentzian profile is similar to the piecewise models used in previous works (e.g., Díaz et al. 2002, 2010; Terradas et al. 2008; Soler et al. 2010). On the contrary, in the parabolic profile the dense prominence plasma is broadly distributed along the tube. The Gaussian profile represent an intermediate situation between the Lorentzian and parabolic profiles. The actual density variation along prominence threads is probably linked to the process that leads to the formation of prominences and the condensation of the cool and dense plasma. A thermal instability might be involved in that process (see, e.g., Luna et al. 2012). The purpose for choosing these three paradigmatic profiles is to determine the effect of different plasma arrangements on the period ratio of kink oscillations.

On the other hand, since we focus on horizontal threads, the external coronal plasma is assumed uniform for simplicity, namely $\rho_{\mathrm{e}}(z)=\rho_{\mathrm{e}}$. Then, we define the prominence to corona density contrast as the ratio of the internal density at the center of the thread to the external density, namely $\zeta=\rho_{\mathrm{i}, 0} / \rho_{\mathrm{e}}$.

The linear ideal MHD equations for a $\beta=0$ plasma that govern the behavior of small perturbations superimposed on the equilibrium prominence thread are

$\rho_{0} \frac{\partial v}{\partial t}=\frac{1}{\mu}(\nabla \times \boldsymbol{b}) \times \boldsymbol{B}$,

$\frac{\partial \boldsymbol{b}}{\partial t}=\nabla \times(\boldsymbol{v} \times \boldsymbol{B})$,

where $\boldsymbol{v}=\left(v_{r}, v_{\varphi}, v_{z}\right)$ and $\boldsymbol{b}=\left(b_{r}, b_{\varphi}, b_{z}\right)$ are the velocity and magnetic field perturbations, respectively, and $\mu$ is the magnetic permeability. Because of the $\beta=0$ approximation, $v_{z}=0$. Since the equilibrium is invariant in the azimuthal direction, we can put the perturbations proportional to $\exp (\operatorname{im} \varphi)$, where $m$ is the azimuthal wavenumber. We consider kink oscillations, so we set $m=1$. In addition, we perform a normal mode analysis and express the temporal dependence of the perturbations as $\exp (-\mathrm{i} \omega t)$, where $\omega$ is the frequency. The period of the oscillation is $P=2 \pi / \omega$. Then, Eqs. (5) and (6) become

$\omega v_{r}=\frac{B_{0}}{\mu \rho_{0}}\left(\frac{\partial b_{r}^{*}}{\partial z}-\frac{\partial b_{z}^{*}}{\partial r}\right)$,

$\omega v_{\varphi}^{*}=\frac{B_{0}}{\mu \rho_{0}}\left(\frac{b_{z}^{*}}{r}-\frac{\partial b_{\varphi}}{\partial z}\right)$

$\omega b_{r}^{*}=-B_{0} \frac{\partial v_{r}}{\partial z}$,

$\omega b_{\varphi}=B_{0} \frac{\partial v_{\varphi}^{*}}{\partial z}$

$\omega b_{z}^{*}=B_{0}\left(\frac{\partial v_{r}}{\partial r}+\frac{v_{r}}{r}+\frac{v_{\varphi}^{*}}{r}\right)$,

where, with no loss of generality, we defined the new perturbations $v_{\varphi}^{*}=\mathrm{i} v_{\varphi}, b_{r}^{*}=\mathrm{i} b_{r}$, and $b_{z}^{*}=\mathrm{i} b_{z}$ in order to remove complex numbers and so to have equations with real coefficients. This is computationally convenient. Physically, the factor $i$ accounts for a phase difference of $\pi / 2$. Therefore, the new perturbations $v_{\varphi}^{*}$, $b_{r}^{*}$, and $b_{z}^{*}$ are $\pi / 2$ out of phase with respect to the actual perturbations $v_{\varphi}, b_{r}$, and $b_{z}$. The remaining perturbations, namely $v_{r}$ and $b_{\varphi}$, are not altered. From here on, we drop the superscript $*$ for simplicity. Equations (7)-(11) define a 2D generalized eigenvalue problem, where $\omega$ is the eigenvalue and the perturbations form the eigenvector. Contrary to the piecewise constant models frequently used in the literature (e.g., Dymova \& Ruderman 2005; Soler et al. 2010; Díaz et al. 2010), the analytic solution to the eigenvalue problem becomes very complicated when $\rho_{0}$ is an arbitrary function of $r$ and $z$ (see Andries et al. 2005b). In this work, the 2D eigenvalue problem is numerically solved with the PDE2D code (Sewell 2005), a general-purpose partial differential equation solver. The PDE2D code was previously used to 
solve a similar 2D eigenvalue problem by Arregui et al. (2011). The PDE2D code uses a collocation method, and the generalized matrix eigenvalue problem is solved using the shifted inverse power method. The code uses finite elements and allows the use of nonuniformly distributed grids. Different grid resolutions have been tested so as to assure the proper convergence of the solutions. The code provides the closest eigenvalue to an initially provided guess and the corresponding spatial form of the eigenfunctions. The boundary conditions used in the code are those consistent with trapped and standing kink oscillations. The two nonzero components of the velocity perturbation, namely $v_{r}$ and $v_{\varphi}$, and the longitudinal component of the magnetic field perturbation, namely $b_{z}$, vanish at $z= \pm L / 2$ because of the linetying boundary condition at the photosphere. In turn, $b_{r}$ and $b_{\varphi}$ have vanishing longitudinal derivatives at $z= \pm L / 2$. In the radial direction, $v_{r}, v_{\varphi}, b_{r}$, and $b_{\varphi}$ have vanishing radial derivatives and $b_{z}=0$ at the axis of the tube, $r=0$. The code self-consistently connects the internal perturbations with the external perturbations at $r=R$ and satisfies the jump relations that naturally arise from the integration of the equations across $r=R$. The condition that the modes are trapped imposes that all perturbations vanish far away from the tube in the radial direction, i.e., when $r \rightarrow \infty$. This condition is accomplished in the numerical code by setting all perturbations equal to zero at $r=r_{\max }$, where $r_{\max }$ is the largest value of the radial coordinate considered in the numerical domain. Ideally, we should set $r_{\max } \rightarrow \infty$. However, this is not possible computationally, and $r_{\max }$ must be set to a finite value. We locate $r_{\max }$ sufficiently far from the tube boundary to properly compute the drop-off rate of perturbations in the radial direction and so avoid numerical errors. To make sure that $r_{\max }$ is sufficiently large, we have performed convergence tests by increasing $r_{\max }$ until the solutions showed no dependence on this parameter. We took $r_{\max }=20 R$ in the computations we include in this paper. In summary, the boundary conditions used in the numerical code are

$$
\begin{aligned}
& \frac{\partial v_{r}}{\partial r}=\frac{\partial v_{\varphi}}{\partial r}=\frac{\partial b_{r}}{\partial r}=\frac{\partial b_{\varphi}}{\partial r}=0, \quad b_{z}=0, \quad \text { at } \quad r=0, \\
& v_{r}=v_{\varphi}=b_{r}=b_{\varphi}=b_{z}=0, \quad \text { at } r=r_{\max } \text {, } \\
& v_{r}=v_{\varphi}=b_{z}=0, \quad \frac{\partial b_{r}}{\partial z}=\frac{\partial b_{\varphi}}{\partial z}=0, \quad \text { at } \quad z= \pm L / 2 \text {. }
\end{aligned}
$$

In the specific case that both the internal and external densities are uniform, namely $\rho_{\mathrm{i}}(z)=\rho_{\mathrm{i}}$ and $\rho_{\mathrm{e}}(z)=\rho_{\mathrm{e}}$, it is possible to derive analytic expressions for the period of kink oscillations in the thin tube (TT) limit, i.e., when $L / R \gg 1$ (see, e.g., Edwin \& Roberts 1983; Goossens et al. 2009). The period of the fundamental longitudinal mode, $P_{0}$, and that of the first longitudinal overtone, $P_{1}$, are given by

$$
P_{0}=\frac{2 L}{v_{\mathrm{A}, \mathrm{i}}} \sqrt{\frac{1+\zeta}{2 \zeta}}, \quad P_{1}=\frac{L}{v_{\mathrm{A}, \mathrm{i}}} \sqrt{\frac{1+\zeta}{2 \zeta}},
$$

where $v_{\mathrm{A}, \mathrm{i}}=B_{0} / \sqrt{\mu \rho_{\mathrm{i}}}$ is the internal Alfvén velocity. Thus, the period ratio is $P_{0} / P_{1}=2$ in longitudinally homogeneous thin tubes. In the present model, deviations from $P_{0} / P_{1}=2$ can be caused by dispersion when the thickness of the thread does not satisfy the TT condition and by longitudinal variation of density. The departure from the TT limit is not likely to have a strong impact for prominence threads, since observations clearly show that threads are very thin and long structures (see Lin 2011). Although only the part of the tubes filled with the densest and coolest plasma can be seen in, e.g., $\mathrm{H} \alpha$ and $\mathrm{Ca}$ II observations, the length of the whole magnetic tube, $L$, must be much longer than the observed length of the prominence threads, $L_{\mathrm{obs}}$. The values of $R$ and $L_{\mathrm{obs}}$ reported by the observations (e.g., Okamoto et al. 2007; Lin et al. 2008; Lin 2011) are in the ranges $50 \mathrm{~km} \lesssim R \lesssim 300 \mathrm{~km}$ and $3000 \mathrm{~km} \lesssim L_{\mathrm{obs}} \lesssim 28000 \mathrm{~km}$, respectively, while Okamoto et al. (2007) estimated $L$ to be at least $L \sim 10^{5} \mathrm{~km}$. These numbers give $10 \lesssim L_{\text {obs }} / R \lessgtr 400$ and $400 \lesssim L / R \lessgtr 2000$, meaning that real prominence threads are actually very thin tubes. Therefore, in the remainder of this paper we shall focus on the effect of longitudinal density variation, which may have a much stronger impact on the period ratio.

\section{Results}

We start by investigating the modification of the fundamental mode and first overtone periods when the ratio of the central density to footpoint density, $\chi$, is increased from $\chi=1$ (homogeneous tube) to $\chi=100$. This is achieved by reducing the density at the footpoints while the central density is kept fixed. These results are displayed in Fig. 3. We denote by $P_{0}^{\prime}$ and $P_{1}^{\prime}$ the fundamental mode and first overtone periods, respectively, numerically computed with the PDE2D code. In turn, $P_{0}$ and $P_{1}$ are the periods of a homogeneous tube with $\rho_{\mathrm{i}}=\rho_{\mathrm{i}, 0}$ computed from Eq. (15).

When $\chi$ increases, both the fundamental mode (Fig. 3a) and first overtone (Fig. 3 b) periods decrease with respect to the values for a homogeneous tube with a density equal to the central density since, in average, the internal density is reduced. The decrease of the periods depends on the specific density profile considered. In this regard, the parabolic profile produces the smallest decrease of the periods. The results for the parabolic profile saturate at $\chi \approx 10$ and become independent of $\chi$ afterwards. On the contrary, the Lorentzian profile produces the largest decrease of the periods, which keep decreasing as $\chi$ increases. The results for the Gaussian profile are in between those of the Lorentzian and parabolic profiles.

Figure $3 \mathrm{c}$ shows the dependence on $\chi$ of the numerically computed period ratio $P_{0}^{\prime} / P_{1}^{\prime}$. Consistently, $P_{0}^{\prime} / P_{1}^{\prime}=2$ when $\chi=1$. As $\chi$ increases, $P_{0}^{\prime} / P_{1}^{\prime}$ takes values larger than 2 . This result conceptually agrees with that obtained by Díaz et al. (2010) in tubes with a piecewise constant density profile. Here, we find that $P_{0}^{\prime} / P_{1}^{\prime}>2$ in the case of continuous density profiles as well. Again, how much the period ratio differs from 2 depends on the density profile considered. The Lorentzian profile produces a significant increase of the period ratio, so that $P_{0}^{\prime} / P_{1}^{\prime} \approx 3.5$ when $\chi=100$. Conversely, the increase of the period ratio is small for the parabolic profile and $P_{0}^{\prime} / P_{1}^{\prime} \approx 2.2$ when $\chi=100$. Finally, we see again that the results for the Gaussian profile are in between those of the Lorentzian and parabolic profiles, so that the period ratio increases moderately with $\chi$ for Gaussian profile and is $P_{0}^{\prime} / P_{1}^{\prime} \approx 2.5$ when $\chi=100$.

It should be noted that comparing the influence of the various profiles using the results of Fig. 3 is not straightforward. When the ratio of the central density to footpoint density is fixed, the total mass in the thread is different for every profile. This fact makes a direct comparison between profiles unsuitable. To fairly compare the results for the various profiles, the total mass in the thread should be the same, which is equivalent to considering the same average density in the thread (see Andries et al. 2005a). Hence, we define the average internal density as

$$
\left\langle\rho_{\mathrm{i}}\right\rangle=\frac{1}{L} \int_{-L / 2}^{L / 2} \rho_{\mathrm{i}}(z) \mathrm{d} z
$$


(a)

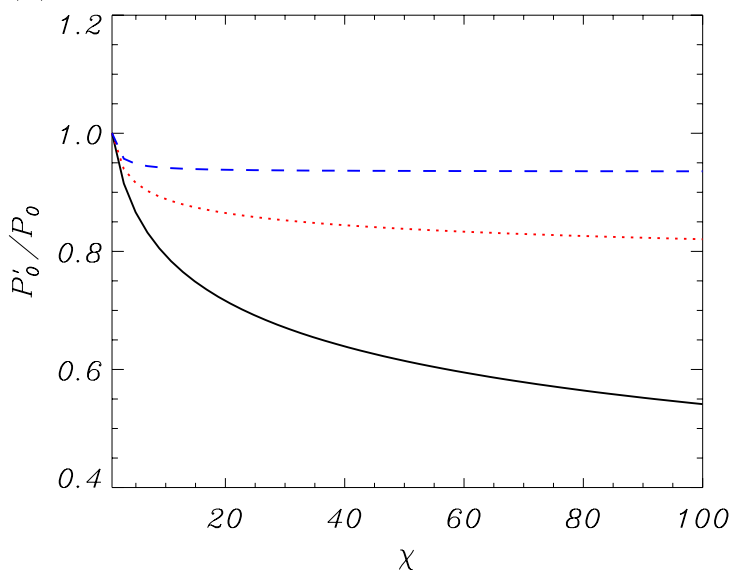

(b)

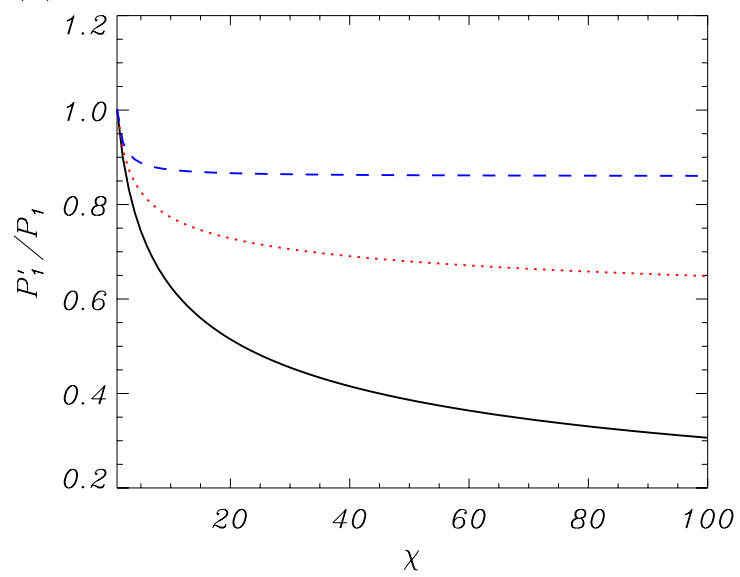

(c)

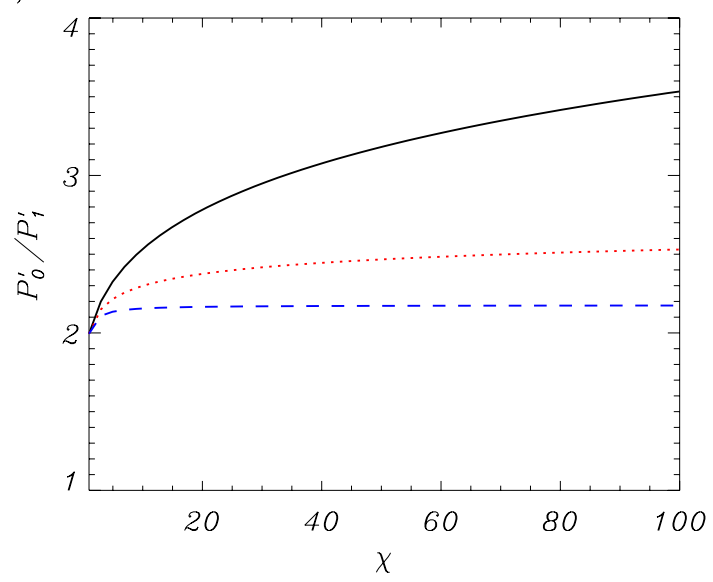

Fig. 3. Dependence of the numerically computed a) fundamental mode period, $P_{0}^{\prime}$, b) first overtone period, $P_{1}^{\prime}$, and c) period ratio, $P_{0}^{\prime} / P_{1}^{\prime}$, with $\chi$ for the Lorentzian profile (black solid line), the Gaussian profile (red dotted line), and the parabolic profile (blue dashed line). We used $L / R=100$ and $\zeta=100$ in all cases. $P_{0}$ and $P_{1}$ denote the periods of a homogeneous thread with $\rho_{\mathrm{i}}=\rho_{\mathrm{i}, 0}$.

Figure 4 displays the same results as Fig. 3, but now $\left\langle\rho_{\mathrm{i}}\right\rangle / \rho_{\mathrm{i}, 0}$ replaces $\chi$ in the horizontal axes of the plots. We see that there are no significant differences between the curves for the various profiles when the average internal density is the same. Importantly, Fig. $4 \mathrm{c}$ shows that the behavior of the period ratio is the same for the three profiles. The period ratio increases as $\left\langle\rho_{\mathrm{i}}\right\rangle / \rho_{\mathrm{i}, 0}$ decreases. This result indicates that the ratio of the average internal density to the central density, namely $\left\langle\rho_{\mathrm{i}}\right\rangle / \rho_{\mathrm{i}, 0}$, and not the ratio of the central density to the footpoint density, namely $\chi$, is the (a)

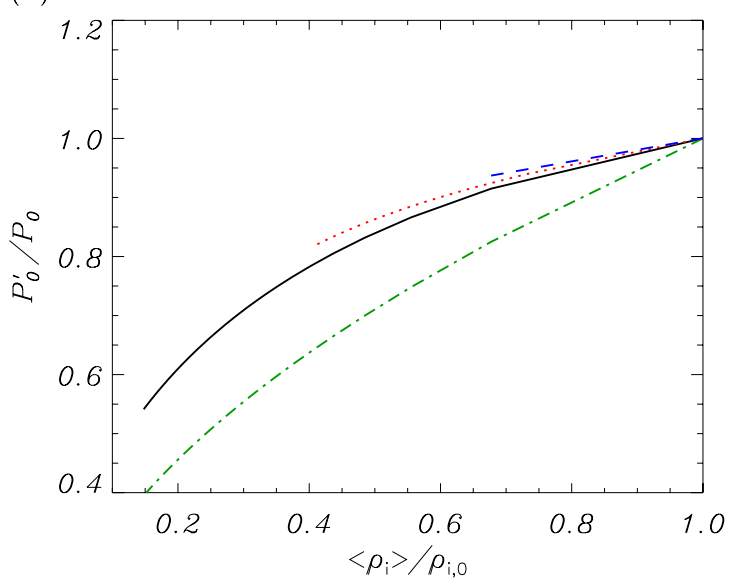

(b)

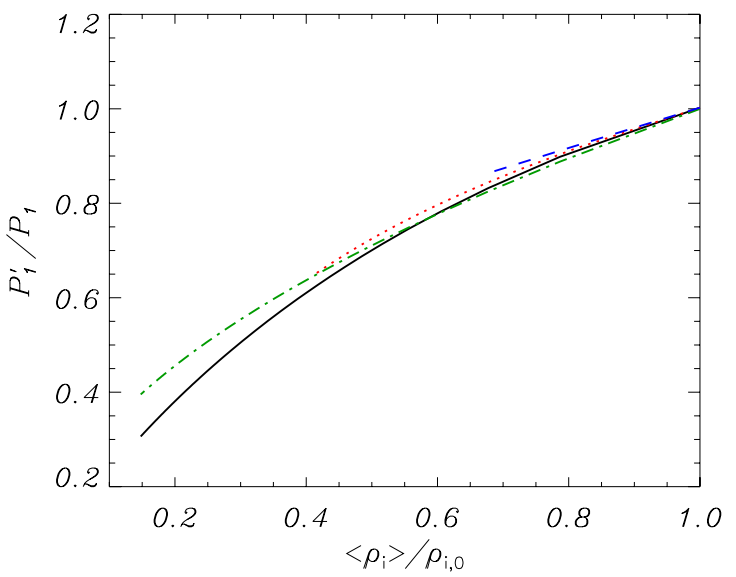

(c)

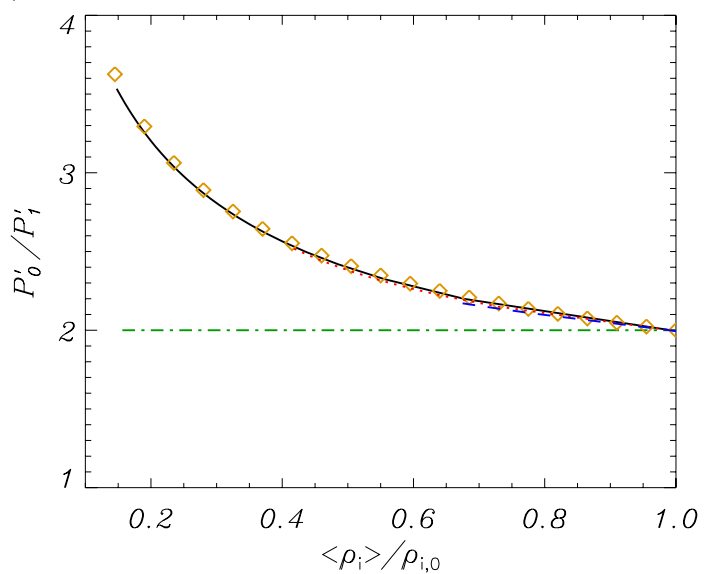

Fig. 4. Same results as Fig. 3 but as a function of the ratio of the average internal density to the central density, $\left\langle\rho_{\mathrm{i}}\right\rangle / \rho_{\mathrm{i}, 0}$. For comparison, the green dash-dotted line shows the result for a homogeneous thread with $\rho_{\mathrm{i}}=\left\langle\rho_{\mathrm{i}}\right\rangle$, i.e., the average thread. The symbols in panel c) correspond to the empirical fit of Eq. (17).

parameter that actually controls the behavior of the period ratio regardless of the form of the density spatial variation along the thread (see Andries et al. 2005a, for an equivalent result in the case of coronal loops). We performed an empirical fit to the results of Fig. 4c and obtained that the dependence of the period ratio on the average density is very closely approximated by

$\frac{P_{0}^{\prime}}{P_{1}^{\prime}} \approx 1+\left(\frac{\left\langle\rho_{\mathrm{i}}\right\rangle}{\rho_{\mathrm{i}, 0}}\right)^{-1 / 2}$, 


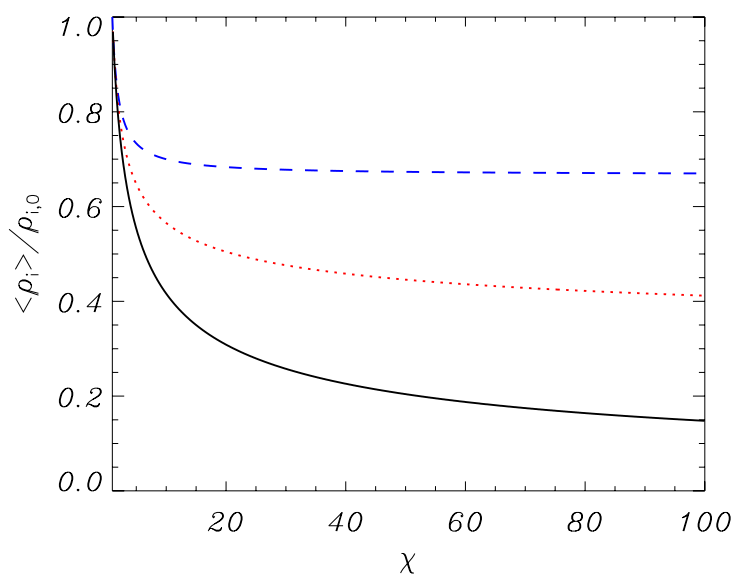

Fig. 5. Average internal density, $\left\langle\rho_{\mathrm{i}}\right\rangle / \rho_{\mathrm{i}, 0}$, as a function of $\chi$. The various lines are for the Lorentzian profile (black solid line), the Gaussian profile (red dotted line), and the parabolic profile (blue dashed line).

for all the profiles. The empirical Eq. (17) is overploted in Fig. 4c to show that the fit is excellent. Equation (17) is independent of the density profile. It is an important result that has simple and straightforward applications for prominence seismology. The implications of this result for prominence seismology are discussed in Sect. 4.

We note that the length of the curves in Fig. 4 is different for different density profiles. The reason for this is that $\left\langle\rho_{\mathrm{i}}\right\rangle$ was computed by varying $\chi$ in the interval $[1,100]$, but the dependence of the average density on $\chi$ is different for every profile. The analytic result of computing the average density from Eq. (16) is

$\left\langle\rho_{\mathrm{i}}\right\rangle=\rho_{\mathrm{i}, 0} \frac{\arctan \sqrt{\chi-1}}{\sqrt{\chi-1}}$,

for the Lorentzian profile,

$\left\langle\rho_{\mathrm{i}}\right\rangle=\rho_{\mathrm{i}, 0} \frac{\sqrt{\pi}}{2} \frac{\operatorname{erf} \sqrt{\log \chi}}{\sqrt{\log \chi}}$,

for the Gaussian profile (where erf and log denote the error function and the natural logarithm, respectively), and

$\left\langle\rho_{\mathrm{i}}\right\rangle=\rho_{\mathrm{i}, 0} \frac{2 \chi+1}{3 \chi}$,

for the parabolic profile. These average densities are plotted in Fig. 5 as a function of $\chi$. We see that the average density of the Lorentzian profile is lower than that of the parabolic profile for the same value of $\chi$, with that of the Gaussian profile in between. For prominence threads it is expected $\chi$ to be a large parameter. Hence, we perform the limit $\chi \gg 1$ and the expressions for the average density of the different profiles simplify to

$\left\langle\rho_{\mathrm{i}}\right\rangle \approx \rho_{\mathrm{i}, 0} \frac{\pi}{2} \chi^{-1 / 2}$,

for the Lorentzian profile,

$\left\langle\rho_{\mathrm{i}}\right\rangle \approx \rho_{\mathrm{i}, 0} \frac{\sqrt{\pi}}{2}(\log \chi)^{-1 / 2}$

for the Gaussian profile, and

$\left\langle\rho_{\mathrm{i}}\right\rangle \approx \rho_{\mathrm{i}, 0} \frac{2}{3}$, for the parabolic profile. Importantly, the average density of the parabolic profile becomes independent of $\chi$ when $\chi \gg 1$. Now we substitute these average densities into Eq. (17) to obtain simple relations between $P_{0}^{\prime} / P_{1}^{\prime}$ and $\chi$ for the various profiles, namely

$\frac{P_{0}^{\prime}}{P_{1}^{\prime}} \approx 1+\left(\frac{4}{\pi^{2}} \chi\right)^{1 / 4}$,

for the Lorentzian profile,

$\frac{P_{0}^{\prime}}{P_{1}^{\prime}} \approx 1+\left(\frac{4}{\pi} \log \chi\right)^{1 / 4}$,

for the Gaussian profile, and

$\frac{P_{0}^{\prime}}{P_{1}^{\prime}} \approx 1+\sqrt{\frac{3}{2}} \approx 2.22$,

for the parabolic profile. These approximate results agree well with the numerical solutions displayed in Fig. 3 when $\chi \gg 1$.

For comparison, we overplot in Fig. 4 the results for a longitudinally homogeneous thread whose internal density is equal to the average density, i.e., $\rho_{\mathrm{i}}=\left\langle\rho_{\mathrm{i}}\right\rangle$. We call this hypothetical case the "average thread". Obviously, the period ratio of the average thread is 2 because of the absence of longitudinal inhomogeneity. However, we see that the fundamental mode period of the average thread is shorter than the period of a nonuniform thread, but the first overtone periods are similar in both cases. The fact that the density varies spatially is important for the fundamental mode period, but is not very relevant for the first overtone period. This points out that ignoring the longitudinal variation of density in prominence threads by using average thread models may provide inaccurate values of the fundamental mode period. For coronal loops, Andries et al. (2005a) showed that the first overtone was more affected by plasma stratification than the fundamental mode. For prominence threads, we find that the fundamental mode is more affected by longitudinal inhomogeneity than the first overtone. Our result is opposite to that found by Andries et al. (2005a), probably because in prominence threads the densest plasma is located near the tube center, while in stratified coronal loops the plasma is denser near the footpoints. We also note that, contrary to Andries et al. (2005a), we do not perform a weighted spatial average of the density but an unweighed average.

Finally, for completeness we have computed the spatial form of the eigenfunctions with the PDE2D code. We display in Fig. 6 longitudinal cuts of $v_{r}, v_{\varphi}$, and $b_{z}$ near the boundary of the thread, i.e., $r \approx R$, for both the fundamental mode and the first overtone using a particular set of parameters. Figure 6 may be compared with the eigenfunctions in piecewise-like models (Soler et al. 2010; Arregui et al. 2011). We can observe in Fig. 6 that the eigenfunctions of the fundamental longitudinal mode of transverse kink waves (left panels) have no nodes in the axial (longitudinal) direction, while the eigenfunctions of the first longitudinal overtone of transverse kink waves (right panels) have one node at the center of the tube. We find that $v_{r}$ and $v_{\varphi}$ corresponding to the different profiles are very similar for both fundamental mode and first overtone. This suggests that measurements of the thread transverse velocity or, equivalent, the displacement could provide little information about the density profile. However, the spatial form of $b_{z}$ is sensitive to the density profile. The more concentrated the dense prominence plasma near the center of the tube, the faster the amplitude of $b_{z}$ drops from the center. This 
(a)

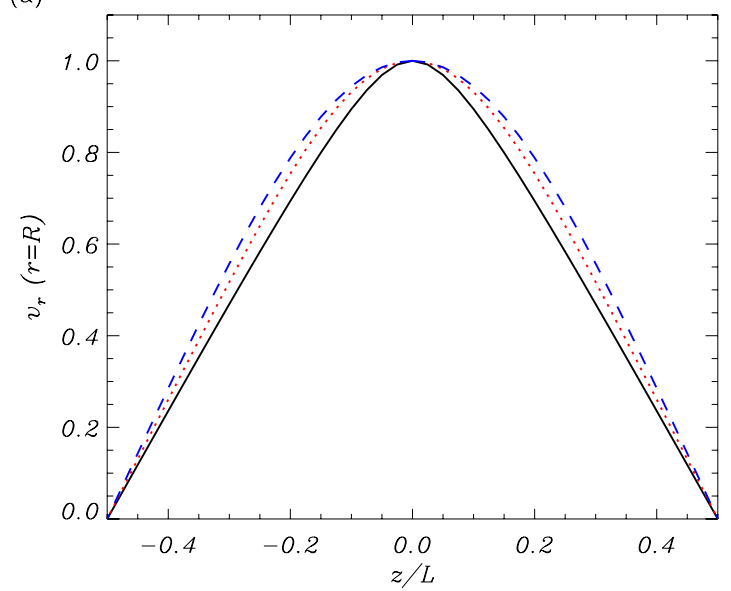

(c)

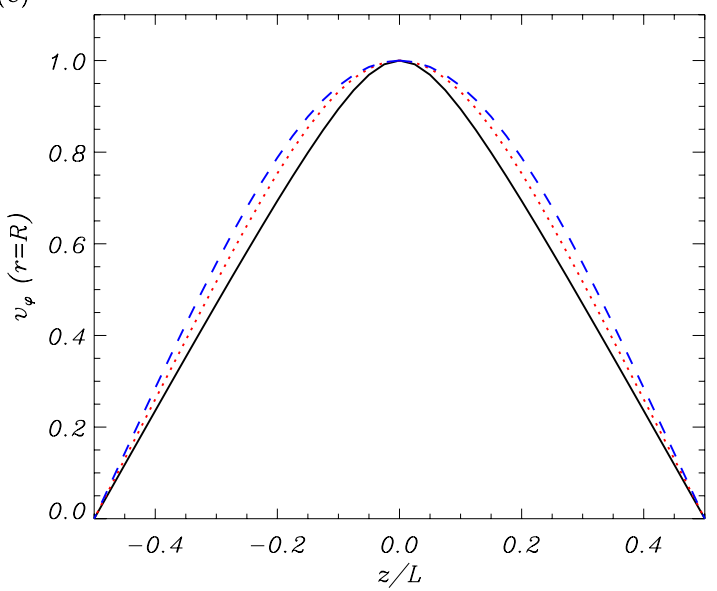

(e)

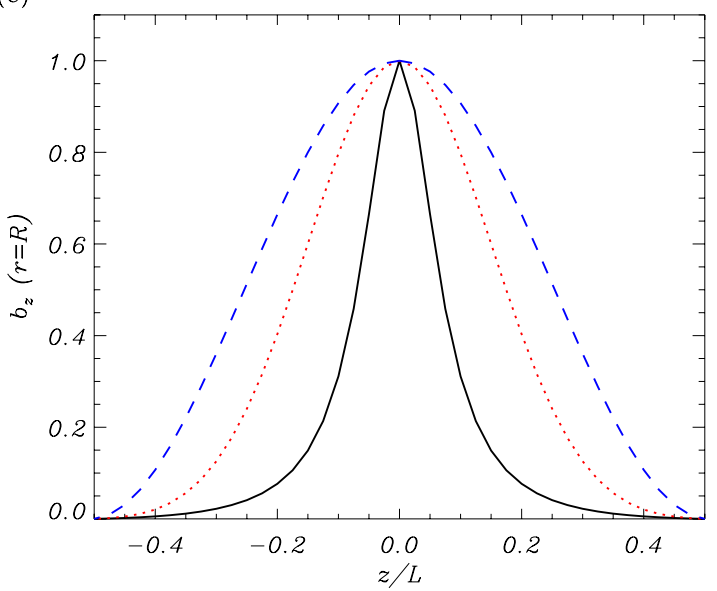

(b)

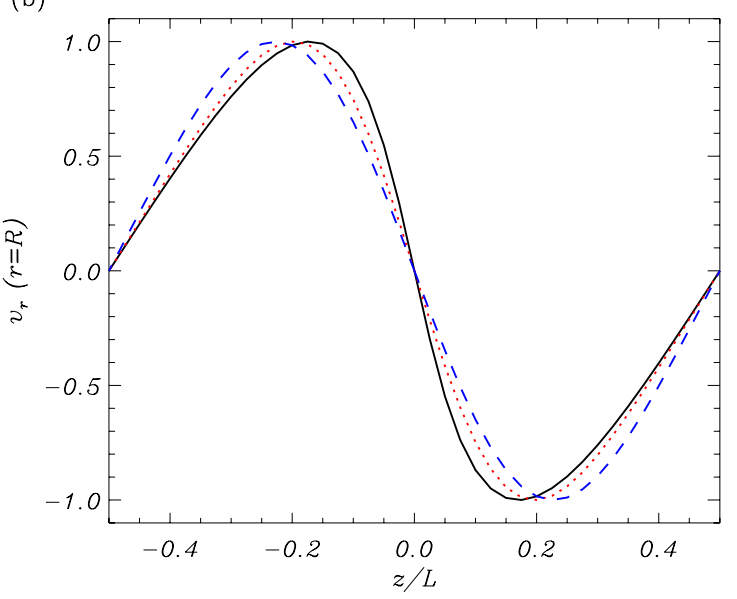

(d)

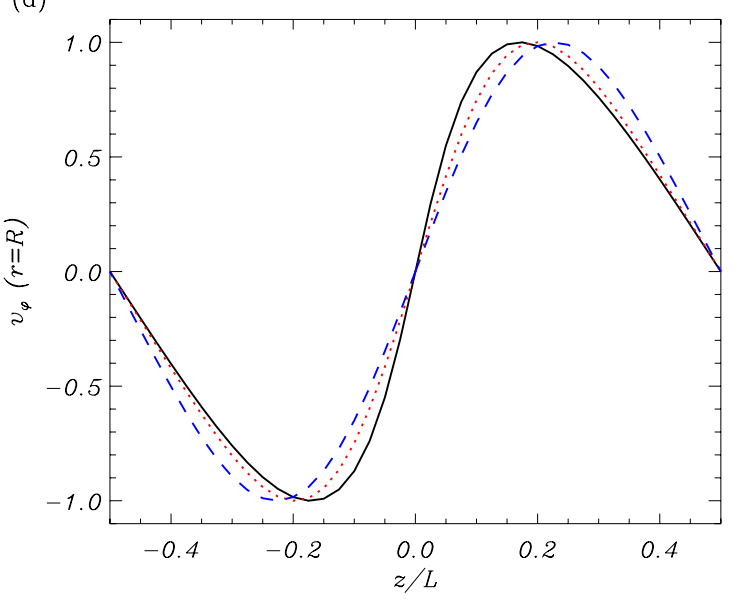

(f)

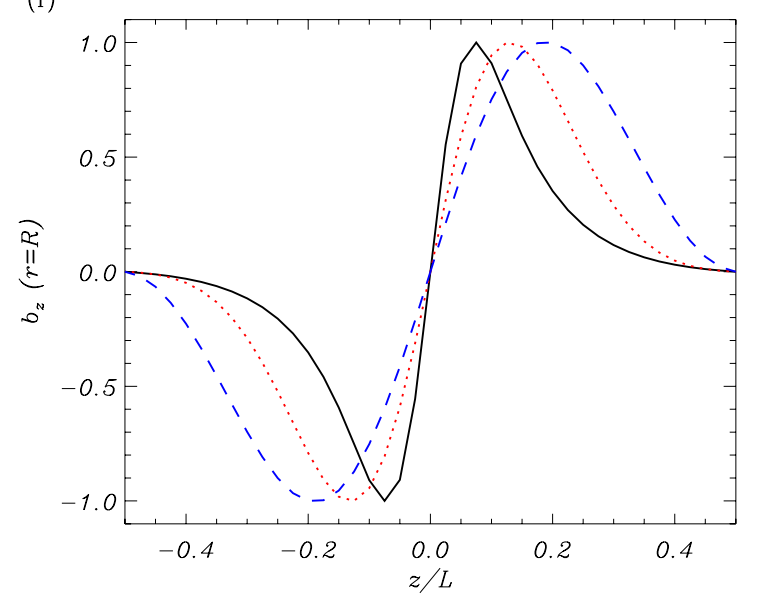

Fig. 6. Longitudinal cuts at $r=R$ of $v_{r}$ (top panels), $v_{\varphi}$ (mid panels), and $b_{z}$ (bottom panels) corresponding to the fundamental mode (left panels) and the first overtone (right panels). The various lines are for the Lorentzian profile (black solid line), the Gaussian profile (red dotted line), and the parabolic profile (blue dashed line). We used $L / R=100, \chi=50$, and $\zeta=100$ in all cases. Arbitrary units are used.

result was also found by Arregui et al. (2011, see their Fig. 6c). In the present computations, $b_{z}$ is essentially confined near the center of the thread for the Lorentzian profile, but it is almost a sinusoidal-type function for the parabolic profile.

\section{Prominence seismology}

The results of this paper and, in particular, the empirical fit of Eq. (17) have important implications for seismology of prominences. Equation (17) is general and does not depend on the specific density profile. This means that it can be used even when the density profile is not known. By inverting Eq. (17), we find that the ratio of the average internal density to the central density is related to the period ratio as

$\frac{\left\langle\rho_{\mathrm{i}}\right\rangle}{\rho_{\mathrm{i}, 0}} \approx\left(\frac{P_{0}^{\prime}}{P_{1}^{\prime}}-1\right)^{-2}$.

Equation (27) can be used to seismologically estimate the ratio $\left\langle\rho_{\mathrm{i}}\right\rangle / \rho_{\mathrm{i}, 0}$ in prominence threads using observations of the period ratio. 
As mentioned in the Introduction, no reliable simultaneous observations of the two periods in prominence threads are currently available. Two periods were reported by Lin et al. (2007). These authors studied an extensive area in a prominence and it is unclear whether the two periods originate from the same thread or correspond to different threads. However, to perform a simple exemplifying application, we proceed as in Díaz et al. (2010) and assume that the two periods reported by Lin et al. (2007) actually correspond to the fundamental mode and the first overtone of the same oscillating thread. The two periods are $P_{0}^{\prime}=16 \mathrm{~min}$ and $P_{1}^{\prime}=3.6 \mathrm{~min}$, which give $P_{0}^{\prime} / P_{1}^{\prime} \approx 4.44$. Then, using Eq. (27) we get $\left\langle\rho_{\mathrm{i}}\right\rangle / \rho_{\mathrm{i}, 0} \approx 0.084$. This result indicates that there is a strong density gradient along the thread, since the average density is a small fraction of the central density. This is compatible with a large value of the ratio of the central density to the footpoint density, i.e., $\chi \gg 1$.

Next, we can also use the observed $P_{0}^{\prime} / P_{1}^{\prime}$ to infer the ratio of the central density to the footpoint density, $\chi$. To do so, we must necessarily assume a particular density profile along the thread. We note that the form of the density profile was not needed to estimate $\left\langle\rho_{\mathrm{i}}\right\rangle / \rho_{\mathrm{i}, 0}$. In the case of the Lorentzian profile we get from Eq. (24) that

$\chi \approx \frac{\pi^{2}}{4}\left(\frac{P_{0}^{\prime}}{P_{1}^{\prime}}-1\right)^{4} \approx 347$,

while for the Gaussian profile we get from Eq. (25) that

$\chi \approx \exp \left[\frac{\pi}{4}\left(\frac{P_{0}^{\prime}}{P_{1}^{\prime}}-1\right)^{4}\right] \approx 10^{48}$.

The value of $\chi$ corresponding to the Gaussian profile is unrealistically large, while the value corresponding to the Lorentzian profile is reasonably consistent with the expected densities in prominence threads. In the case of the parabolic profile, we found in Eq. (26) that its period ratio is 2.22 when $\chi \gg 1$, so that the observed period ratio of 4.44 seems hardly compatible with a parabolic profile. The inferred values of $\chi$ suggest that, among the three profiles studied here, the Lorentzian profile may provide the better explanation for the ratio of the two periods reported by Lin et al. (2007). However, a rigorous comparison between models should be done and an adequate treatment of errors should be considered to obtain more robust conclusions (see Arregui et al. 2013).

\section{Concluding remarks}

In this paper, we have investigated the ratio of the period of the fundamental mode to period of the first overtone of kink oscillations of longitudinally inhomogeneous prominence threads. Contrary to previous works that used piecewise constant density profiles (Díaz et al. 2010), here we assumed a continuous nonuniform density profile along the thread, which is more consistent with the simulations of thread formation via plasma condensation in solar prominences (e.g., Luna et al. 2012). Our results indicate that the ratio of the average internal density to the central density, namely $\left\langle\rho_{\mathrm{i}}\right\rangle / \rho_{\mathrm{i}, 0}$, is the parameter that controls the behavior of the period ratio regardless of the form of the density spatial variation along the thread. A fit of the period ratio as a function of $\left\langle\rho_{\mathrm{i}}\right\rangle / \rho_{\mathrm{i}, 0}$ was obtained, which can be used to estimate the degree of nonuniformity along the threads if observations of the two periods are reported. From the observational point of view, the detection of the first overtone is challenging, since the amplitude in the densest part of the tube is small for that mode. Although difficult, we believe that the observation could be done with present or future high-resolution instruments. For instance, using recent observations with the Hinode satellite, Hillier et al. (2013) reported a large number of oscillations in vertical prominence threads with velocity amplitudes as low as $0.2 \mathrm{~km} \mathrm{~s}^{-1}$. In view of these observations, the detection of oscillations with such small amplitudes in horizontal threads is more than likely.

Here we have studied the period ratio from a purely numerical point of view. Hence, the equation that relates the period ratio with the average density (Eq. (17)) was empirically obtained from the numerical data. Although it is beyond the purpose of the present study, it would be desirable that future works pursue a more robust theoretical, i.e., analytical, explanation for this relation. The analytical theory developed by Dymova \& Ruderman (2005) could be used to do so.

In the model, we assumed for simplicity that the density within the flux tube is symmetric with respect to $z=0$. The effect of shifting the density enhancement from the tube center was investigated in a previous paper by Soler et al. (2010) using a simple piecewise model. The conclusion from Soler et al. (2010) was that the periods of kink oscillations are weakly affected by the position of the densest plasma within the tube unless the density enhancement is put very close to the footpoints, which is not realistic in the case of horizontal prominence threads. Therefore, we expect that the use of an asymmetric density profile would not change significantly the results of the present paper.

The results of the seismology application of Sect. 4 suggest that value of the period ratio could be used to distinguish between different density profiles. In a general sense, rigorous statistical methods to perform model comparison, as the Bayesian analysis used by Arregui et al. (2013), could be very useful to determine what kind of density variations are the most probable ones for a given value of the period ratio. Future works should exploit this possibility. Furthermore, the presence of uncertainties in the measurements of the periods should be properly accounted for.

Finally, we should mention that there are some effects not included in the prominence thread model that may influence the value of the period ratio. For instance, an ingredient missing from the model is the presence of mass flows along the thread. Simultaneous transverse oscillations and flows in prominence fine structures have been observed (e.g., Okamoto et al. 2007). The effect of flows on the period ratio has been investigated in piecewise constant models (e.g., Soler \& Goossens 2011; Erdélyi et al. 2014), but not in threads with nonuniform density profiles. Among other effects, the roles of magnetic field twist, curvature, and cross section have been studied in the case of coronal loops (see, e.g., Verth \& Erdélyi 2008; Verth et al. 2008; Ruderman et al. 2008; Morton \& Erdélyi 2009; Karami \& Bahari 2012) but they should be explored for prominence threads as well. In addition, in view of the large number of observations (see, e.g., Hillier et al. 2013), the problem of the transverse oscillations of vertical threads is also theoretically relevant. For vertical threads, the effect of gravitational stratification should be included in the model. A comparison between the properties of the oscillations of vertical and horizontal threads is an interesting task to carry out in forthcoming works.

Acknowledgements. We thank Iñigo Arregui for reading a draft of this paper and for giving helpful comments. We also thank Teimuraz Zaqarashvili for discussions on the Lomineishvili et al. (2014) paper. We acknowledge support from MINECO and FEDER funds through project AYA2011-22846. R.S. also acknowledges support from CAIB through the "Grups Competitius" program and FEDER funds, from MINECO through a "Juan de la Cierva" grant, from 
MECD through project CEF11-0012, and from the "Vicerectorat d'Investigació i Postgrau" of the UIB. M.G. acknowledges support from KU Leuven via GOA/2009-009 and also partial support from the Interuniversity Attraction Pols programme initiated by the Belgian Science Policy Office (IAP P7/08 Charm).

\section{Appendix A: Comment on the paper by Lomineishvili et al. (2014)}

The results computed in the present paper for a thread with a parabolic density profile are in disagreement with those obtained by Lomineishvili et al. (2014) using the same model. Lomineishvili et al. (2014) claim that the period ratio is 3 , but we found that the correct value of the period ratio is 2.22, approximately. Here we explain the source of the discrepancy between the results of Lomineishvili et al. (2014) and those of the present paper.

A property of the analysis by Lomineishvili et al. (2014) is the discontinuous behavior of their solutions as a function of the inhomogeneity parameter $\alpha$ at $\alpha=0$. The parameter $\alpha$ used by Lomineishvili et al. (2014) is related to our parameter $\chi$ as $\alpha=(\chi-1) / \chi$, so that $\alpha=0$ corresponds to $\chi=1$ in our notation. As Lomineishvili et al. (2014) observe (see the paragraph that follows their Eq. (19)) $\alpha=0$ in their Eq. (17) leads to the solution of the homogeneous tube. They continue to say that after corresponding calculation, one may recover the well-known dispersion relation obtained by Edwin \& Roberts (1983). The ratio of the period of the fundamental longitudinal mode to that of the first overtone for a homogeneous tube in the TT approximation is 2 . For $\alpha \neq 0$, however small the value of $\alpha$ is, Lomineishvili et al. (2014) claim that this ratio is 3 in the TT approximation. According to the analysis by Lomineishvili et al. (2014) the period ratio varies in a discontinuous manner at $\alpha=0$, where it jumps from 2 for $\alpha=0$ to 3 for $\alpha>0$. Since longitudinal density stratification is not a singular perturbation to the problem of linear waves on magnetic cylinders, this result is inconsistent.

Equations (53) and (54) of Lomineishvili et al. (2014) correspond to analytic expressions for the fundamental mode and first overtone frequencies in the TT approximation. Setting $\alpha \rightarrow 0$ in Eqs. (53) and (54) of Lomineishvili et al. (2014) results in vanishing frequencies, i.e., infinite periods. This result can also be seen in the numerically obtained frequencies plotted in their Fig. 3. Lomineishvili et al. (2014) do not recover the frequencies of Edwin \& Roberts (1983) when $\alpha \rightarrow 0$.

According to Zaqarashvili (priv. comm.), the modes obtained by Lomineishvili et al. (2014) owe their existence to inhomogeneity and so they disappear when the thread is homogeneous, i.e., when $\alpha \rightarrow 0$. According to Zaqarashvili (priv. comm.), Lomineishvili et al. (2014) assumed open boundary conditions at the ends of the thread because in their model "the thread itself probably is a part of much longer magnetic tube, which has a coronal density outside the thread". However, fixed boundary conditions are necessary for standing kink oscillations. Hence, the mathematical analysis of Lomineishvili et al. (2014) does not lead to a dispersion relation valid for standing kink oscillations.

\section{References}

Andries, J., Arregui, I., \& Goossens, M. 2005a, ApJ, 624, L57

Andries, J., Goossens, M., Hollweg, J. V., Arregui, I., \& Van Doorsselaere, T. 2005b, A\&A, 430, 1109

Andries, J., van Doorsselaere, T., Roberts, B., et al. 2009, Space Sci. Rev., 149, 3

Arregui, I., Terradas, J., Oliver, R., \& Ballester, J. L. 2008, ApJ, 682, L141 Arregui, I., Soler, R., Ballester, J. L., \& Wright, A. N. 2011, A\&A, 533, A60

Arregui, I., Ballester, J. L., Oliver, R., Soler, R., \& Terradas, J. 2012a, in 4th Hinode Science Meeting: Unsolved Problems and Recent Insights, eds. L. Bellot Rubio, F. Reale, \& M. Carlsson, ASP Conf. Ser., 455, 211

Arregui, I., Oliver, R., \& Ballester, J. L. 2012b, Liv. Rev. Sol. Phys., 9, 2

Arregui, I., Asensio Ramos, A., \& Díaz, A. J. 2013, ApJ, 765, L23

Ballester, J. L. 2014, IAU Symp., 300, 30

Berger, T. E., Shine, R. A., Slater, G. L., et al. 2008, ApJ, 676, L89

Casini, R., López Ariste, A., Tomczyk, S., \& Lites, B. W. 2003, ApJ, 598, L67

Díaz, A. J., Oliver, R., \& Ballester, J. L. 2002, ApJ, 580, 550

Díaz, A. J., Oliver, R., \& Ballester, J. L. 2010, ApJ, 725, 1742

Donnelly, G. R., Díaz, A. J., \& Roberts, B. 2007, A\&A, 471, 999

Dymova, M. V., \& Ruderman, M. S. 2005, Sol. Phys., 229, 79

Dymova, M. V., \& Ruderman, M. S. 2006, A\&A, 459, 241

Edwin, P. M., \& Roberts, B. 1983, Sol. Phys., 88, 179

Erdélyi, R., Hague, A., \& Nelson, C. J. 2014, Sol. Phys., 289, 167

Goossens, M., Terradas, J., Andries, J., Arregui, I., \& Ballester, J. L. 2009, A\&A, 503, 213

Goossens, M., Erdélyi, R., \& Ruderman, M. S. 2011, Space Sci. Rev., 158, 289

Hillier, A., Morton, R. J., \& Erdélyi, R. 2013, ApJ, 779, L16

Karami, K., \& Bahari, K. 2012, ApJ, 757, 186

Lin, Y. 2011, Space Sci. Rev., 158, 237

Lin, Y., Engvold, O., Rouppe van der Voort, L. H. M., \& van Noort, M. 2007, Sol. Phys., 246, 65

Lin, Y., Martin, S. F., Engvold, O., Rouppe van der Voort, L. H. M., \& van Noort, M. 2008, Adv. Space Res., 42, 803

Lin, Y., Soler, R., Engvold, O., et al. 2009, ApJ, 704, 870

Lomineishvili, S. N., Zaqarashvili, T. V., Zhelyazkov, I., \& Tevzadze, A. G. 2014, A\&A, 565, A35

Luna, M., Karpen, J. T., \& DeVore, C. R. 2012, ApJ, 746, 30

McEwan, M. P., Donnelly, G. R., Díaz, A. J., \& Roberts, B. 2006, A\&A, 460, 893

McEwan, M. P., Díaz, A. J., \& Roberts, B. 2008, A\&A, 481, 819

Morton, R. J., \& Erdélyi, R. 2009, A\&A, 502, 315

Ning, Z., Cao, W., Okamoto, T. J., Ichimoto, K., \& Qu, Z. Q. 2009, A\&A, 499 595

Okamoto, T. J., Tsuneta, S., Berger, T. E., et al. 2007, Science, 318, 1577

Orozco Suárez, D., Asensio Ramos, A., \& Trujillo Bueno, J. 2014a, A\&A, 566 A46

Orozco Suárez, D., Díaz, A. J., Asensio Ramos, A., \& Trujillo Bueno, J. 2014b, ApJ, 785, L10

Ruderman, M. S., Verth, G., \& Erdélyi, R. 2008, ApJ, 686, 694

Schmieder, B., Chandra, R., Berlicki, A., \& Mein, P. 2010, A\&A, 514, A68

Sedláček, Z. 1971, J. Plasma Phys., 5, 239

Sewell, G. 2005, The Numerical Solution of Ordinary and Partial Differential Equations (Wiley \& Sons)

Soler, R., \& Goossens, M. 2011, A\&A, 531, A167

Soler, R., Oliver, R., Ballester, J. L., \& Goossens, M. 2009, ApJ, 695, L166

Soler, R., Arregui, I., Oliver, R., \& Ballester, J. L. 2010, ApJ, 722, 1778

Soler, R., Ruderman, M. S., \& Goossens, M. 2012, A\&A, 546, A82

Soler, R., Oliver, R., \& Ballester, J. L. 2014, in IAU Symp. 300, eds. B. Schmieder, J.-M. Malherbe, \& S. T. Wu, 48

Terradas, J., Arregui, I., Oliver, R., \& Ballester, J. L. 2008, ApJ, 678, L153

Terradas, J., Soler, R., Díaz, A. J., Oliver, R., \& Ballester, J. L. 2013, ApJ, 778, 49

Van Doorsselaere, T., Nakariakov, V. M., \& Verwichte, E. 2007, A\&A, 473, 959

Verth, G., \& Erdélyi, R. 2008, A\&A, 486, 1015

Verth, G., Erdélyi, R., \& Jess, D. B. 2008, ApJ, 687, L45 